\title{
On Soft-information-based Error and Erasure Decoding of Reed-Solomon Codes in Burst Rayleigh Fading Channels
}

\author{
Yong Li, Member, IEEE, Xiang Huang, Jiguang He, Hongqing Liu, Senior Member, IEEE, \\ and Trieu-Kien Truong, Life Fellow, IEEE
}

\begin{abstract}
In this paper, two new decoding algorithms to decode Reed-Solomon (RS) codes during transmission over burst Rayleigh fading channels with additive white Gaussian noise (AWGN) are proposed. They only conduct error correction for coded symbols located in the pure AWGN region, and conduct error and erasure correction for those symbols located in the burst fading region by treating those coded symbols that are very likely erroneous as erasures. The first algorithm does not need to know the fading locations in advance, while the second algorithm assumes that the fading locations are known. In addition, the performance of such two algorithms is studied when a precomputed threshold is used to determine the erasures of the code. Simulation results show that our proposed algorithms not only significantly perform better than the classic Berlekamp-Messay (BM) algorithm with a comparable computational complexity, but also achieve a better trade-off between the performance and the computational complexity when compared with other existing algorithms. In particular, our algorithms exhibit excellent robustness for tested various code parameters and fading configurations. Furthermore, a more detailed mathematical analysis is also developed in this paper in order to estimate the performance of the new algorithms in the burst Rayleigh fading channels. We observe that the performance of the first algorithm can only be estimated relatively accurately when encountering burst deep-fading, whereas the performance prediction for the second algorithm is always in agreement with the simulation results for various fading cases.
\end{abstract}

Index Terms-Reed-Solomon codes, Rayleigh fading, burst error, error and erasure decoding.

\section{INTRODUCTION}

$\mathbf{R}$ EED-SOLOMON (RS) codes for correcting both errors and erasures are widely used in many digital communications and recording systems, such as space communication links [1], high definition television (HDTV) [2], digital versatile discs (DVD) [3], and the IEEE 802.16 standard [4]. The conventional unique decoding algorithms such as the Berlekamp-Massey (BM) algorithm [5], [6], the Euclidean algorithm [7], and the Berlekamp-Welch (BW) algorithm [8]

$\mathrm{Y}$. Li is with the College of Computer Science, Chongqing University, Chongqing 400044, China (e-mail: liyongxmu@gmail.com).

$\mathrm{X}$. Huang, and H. Liu are with the Key Lab of Mobile Communication, Chongqing University of Posts and Telecommunications, Chongqing, 400065 China (e-mail: eric.huang.xiang@ foxmail.com, hongqingliu@cqupt.edu.cn).

J. He is the Centre for Wireless Communications, University of Oulu, 90014 Oulu, Finland (e-mail: jiguang.he@oulu.fi).

T.-K. Truong is with the Department of Information Engineering, I-Shou University (e-mail: truong@isu.edu.tw), the Department of Computer Science and Engineering, National Sun Yat-sen University, Kaohsiung City, Taiwan.

This work was supported by China NSF under Grants No. 61771081 and 61501072. (Corresponding author: Yong Li.) are very efficient but the error-correcting capability of the code is limited by the half Hamming distance bound. In contrast, list decoding algorithms attempt to determine the codewords within a specified distance from a received word and hence improve the error-correcting capability of a given code.

Building on the BW algorithm, Sudan [9] discovered a polynomial-time list decoding algorithm. It breaks through the classical error-correcting capability $\left\lfloor\frac{n-k}{2}\right\rfloor$, where $\lfloor x\rfloor$ denotes the largest integer less than or equal to $x$, and $n$ and $k$ denote the code length and the information length of an RS code, respectively. Nevertheless, such an algorithm is effective merely when the code rate is less than $\frac{1}{3}$. For this reason, in 1999, Guruswami and Sudan [10] developed an improved version of Sudan's algorithm, which is capable of correcting more than $\left\lfloor\frac{n-k}{2}\right\rfloor$ errors with a polynomialtime complexity for all code rates. This algorithm consists of two steps: (i) computation of interpolation process, (ii) factorization of a bivariate polynomial. Later, the algorithm in [10] was modified by Koetter and Vardy, see [11], so that the soft-decision reliability information provided by the channel is inserted into the interpolation process. This is called the Koetter-Vardy (KV) algorithm, which achieves a significant gain over the preceding hard-decision (HD) decoding and list decoding algorithms over AWGN channels. However, it is still orders of magnitude higher in complexity than the HD decoding algorithms. As a result, the KV algorithm is not practical.

The two-dimensional interpolation needed in the KV algorithm increases the decoding complexity and hence will be much more time-consuming. Consequently, various simplified interpolation approaches were developed. Among them, early examples of using the coordinate transform of the interpolation points were given by the authors in [12], [13]. Bellorado et al. [14] presented a Chase-type interpolation, which exploits the similarity among test vectors to obtain the set of candidate codewords; thereby reducing the interpolation complexity. $\mathrm{Wu}$ [15] developed a one-pass Chase algorithm for decoding RS codes, which has a complexity of $O\left(d n^{2}\right)$, where $d$ is the minimum distance. In [15], the corresponding VLSI architecture was also designed. Chen et al. [17] proposed a progressive algebraic soft-decision (ASD) decoding algorithm in which the factorization output list size is enlarged progressively.

Clearly, the computational complexity of the ASD algorithms mentioned above is still very high from the implementation point of view, which does not make these algorithms 
particularly suited for storage and deep space communications. Towards this end, Lee et al. [23] proposed a simplified softdecision decoding algorithm of RS codes by constraining the erasure positions according to the soft information and utilizing successive error-and-erasure decoding (EED). They also mathematically estimated the performance based on the ordered statistics of symbol reliability. Simulation results show that the algorithm in [23] exhibits considerable performance gain over the previous soft-decision decoding schemes such as the KV and Chase-II algorithms at a moderate computational complexity. In [18], Ur-Rehman and Zivic proposed a list decoding algorithm for RS codes based on Chase's idea. Such an algorithm erases $\lambda$ symbols with least reliabilities and then performs the EED iteratively by flipping different combinations of $\eta$ least reliable bits, thereby achieving a better trade-off between the performance and the computational complexity when compared with the algorithm in [23].

Actually, RS codes are capable of correcting up to $n-k$ symbol errors with relatively small probabilities of miscorrection if these errors are clustered into bursts. In [19], Chen et al. developed a decoding algorithm for correcting a burst of length greater than $\left\lfloor\frac{n-k}{2}\right\rfloor$ by utilizing syndromes. This algorithm needs to solve a probably singular linear equation system and thus has a high computational complexity. Dawson and Khodkar [20] observed that the formation of key equations and the calculation of the roots of key equations in [19] can be obtained more efficiently in another manner, and developed a simplified method to identify all single-bursts. However, the method is still computationally costly because of having to try different burst lengths. In 2001, Yin et al. [21] proposed a simplified algorithm by utilizing the cyclic property of RS codes. This algorithm avoids the complex division operation by using a cyclic shift method. However, it still requires a computational complexity $O\left(v^{2} n\right)$ where $v \triangleq n-k$. Recently, Wu [22] developed a single-burst correction algorithm with complexity $O(v n)$. Furthermore, Wu generalized this algorithm to the cases in which there are one or several random errors beyond a single burst.

In this paper, we develop an erasure-marking technique in the RS-coded burst Rayleigh fading channels with binary phase shift keying (BPSK) modulation and present two simple soft information based decoding algorithms of RS codes by combining the new erasure-marking scheme and the conventional error-and-erasure decoder. For instance, suppose that there are $b$ bits in a symbol and all the bits are independent. If the error probabilities of some bits in each symbol exceeds a threshold, then that symbol has a high likelihood of being an error and can be treated as an erasure. This leads to extend the error-correcting capability of the code if RS decoders for correcting both errors and erasures are employed. Extensive simulation results show that the new decoding algorithms perform very well while their decoding complexity is comparable with that of the BM algorithm. In particular, the new algorithms exhibit very outstanding robustness for different code parameters and channel characteristics. Additionally, we also analyze the performance of the new algorithms mathematically over burst Rayleigh fading channels, which conforms to the simulation results in the tested signal-to-noise ratio (SNR) regions when the burst fading is deep.

The remainder of this article is organized as follows: The background knowledge of RS codes is reviewed in Section II. Section III demonstrates two methods of correcting errors as well as erasures in which the erasures can be determined by the symbols that have the high likelihood of being errors. Moreover, the corresponding mathematical analysis is also provided in this section. Simulation results are presented in Section IV. Finally, this paper concludes with a brief summary in the final section.

\section{TERminology AND BACKGROUND OF RS CODES}

Let $C$ be a $(n, k)$ RS code over $G F\left(2^{m}\right)$ with minimum distance $d$, where $n<2^{m}$ is the block length, $k$ is the number of $m$-bit message symbols, and $d-1=n-k$ is the number of parity symbols, a property known as maximally distance separable. The maximum number of errors in an RS code which can be corrected is $t=\lfloor(d-1) / 2\rfloor$. The generator polynomial $g(x)$ is of the form

$$
g(x)=\prod_{i=1}^{2 t}\left(x-\alpha^{i}\right)
$$

where $\alpha$ is a primitive element of the finite field $G F\left(2^{m}\right)$. For any message polynomial $u(x)=u_{0}+u_{1} x+\ldots+u_{k-1} x^{k-1}$, the encoded codeword is $c(x)=x^{n-k} u(x)+p(x)$, where $c(x)=c_{0}+c_{1} x+\ldots+c_{n-1} x^{n-1}$ and $p(x)$ is the parity polynomial of degree less than $n-k$, given by

$$
p(x) \equiv x^{n-k} u(x) \bmod g(x) .
$$

Since $c(x)$ is a multiple of $g(x)$, one obtains

$$
c\left(\alpha^{i}\right)=0 \text { for } i=1,2, \ldots, 2 t .
$$

A codeword having $n$ symbols generated in this manner is said to be a systematic codeword.

Suppose an RS codeword is transmitted through a noisy channel. Let $\boldsymbol{r}=\left(r_{0}, r_{1}, \ldots, r_{n-1}\right)$ be the received harddecision vector. Its associated polynomial $r(x)=r_{0}+r_{1} x+$ $\ldots+r_{n-1} x^{n-1}$ can be expressed as the sum of the codeword polynomial $c(x)$ and the error polynomial $e(x)$, where $e(x)=e_{0}+e_{1} x+\ldots+e_{n-1} x^{n-1}$. The following is a brief review of the classical BM decoding algorithm, mentioned in [5], [6]. Initially, a series of syndromes are obtained by evaluating the received polynomial at $2 t$ consecutive locators, given by

$$
S_{i}=r\left(\alpha^{i}\right) \text { for } i=1,2, \ldots, 2 t .
$$

If all the syndromes are equal to zero, then we assume that there were no errors during transmission; otherwise, the BM decoder is used to determine the error locators and error magnitudes.

Now, let us first define the syndrome polynomial $S(x)$, the error-locator polynomial $\Lambda(x)$, and the error-evaluator polynomial $\Omega(x)$ as follows:

$$
\begin{gathered}
S(x)=S_{0}+S_{1} x+\ldots+S_{2 t-1} x^{2 t-1} . \\
\Lambda(x)=\prod_{i=1}^{\delta}\left(1-\alpha_{i} x\right)=1+\Lambda_{1} x+\ldots+\Lambda_{\delta} x^{\delta} .
\end{gathered}
$$




$$
\begin{aligned}
\Omega(x) & =\sum_{i=1}^{\delta} \gamma_{i} \alpha_{i} \prod_{j=1, j \neq i}^{\delta}\left(1-\alpha_{j} x\right) \\
& =\Omega_{0}+\Omega_{1} x+\ldots+\Omega_{\delta-1} x^{\delta-1} .
\end{aligned}
$$

Here, $\gamma_{i}, \alpha_{i}$ are the $i$-th errata amplitude and errata location, respectively, and $\delta$ denotes the number of errors. The above three polynomials jointly satisfy the well-known key equation [26], namely,

$$
\Lambda(x) S(x) \equiv \Omega(x) \bmod x^{2 t} .
$$

The BM algorithm can be applied to solve Eq. (8) if $\delta \leq t$. Another widely used approach to solve the key equation is the Euclidean algorithm, see [7], [28].

When erasures are considered, Eq. (8) needs to be modified as below:

$$
\Lambda(x) \Theta(x) S(x) \equiv \Omega(x) \bmod x^{2 t},
$$

where $\Theta(x)=\prod_{j=1}^{v}\left(1-\beta_{j} x\right)$ is the so-called erasure-locator polynomial, $v$ is the number of erasures and $\beta_{j}$ corresponds to the $j$-th erasure location.

\section{SOFT-DECISION DECODING OVER BURST-FADING AWGN CHANNELS}

\section{A. Two New Soft Information Based Decoding Algorithms}

Consider an RS code of length $n$ with BPSK modulation transmitted over burst-fading (that is, a series of consecutive coded bits encounter fading) AWGN channels. This type of channels can be seen as a special case of two-state hidden Markov model (HMM) channels [32], in which the channel states are partitioned into two cases: without burst-fading and with burst-fading (both states are interfered by AWGN), corresponding to good and bad states in HMM channels, respectively. The received signal is $z(t)=h(t) s(t)+w(t)$, where $s(t)$ is the transmitted signal, $h(t)$ is the channel gain, and $w(t)$ is a zero-mean white Gaussian noise process whose power spectral density is equal to $N_{0} / 2$. In order to detect the $k$-th received bit, $z(t)$ is sent through a demodulator such that it produces a sequence of $N$ numbers, denoted by $z_{1}, z_{2}, \ldots, z_{N}$, where $N=n \times m$. That is,

$$
z_{\xi}=h s_{\xi}+w_{\xi} \text { for } \xi=1,2, \ldots, n m .
$$

In Eq. (10), $z_{\xi}$ denotes the $\xi$-th channel observation and its absolute value is, what is called, bit magnitude hereinbelow, $s_{\xi} \in\{-1,+1\}$ is a transmitted binary digit, and $w_{\xi}$ is a white Gaussian random variable with zero mean and variance $\sigma^{2}$. The parameter $h$ is defined to be

$$
h= \begin{cases}a & \text { for } f_{l} \leq \xi<f_{l}+L_{b}, \\ 1.0 & \text { otherwise }\end{cases}
$$

Here, $a$ is a Rayleigh fading coefficient, $f_{l}$ is the starting point of burst fading and $L_{b}$ is the burst length. We assume that $a$ remains static over a codeword period. The probability density function (PDF) of $a$ is given by

$$
p(a)=\frac{a}{\sigma_{a}^{2}} e^{\frac{-a^{2}}{2 \sigma_{a}^{2}}}, a \geq 0 .
$$

Here, $\sigma_{a}$ is the scale parameter of a Rayleigh distribution.
Proposition 1: Assume a sequence of independent binary random variables is transmitted over the AWGN channel and BPSK modulation is used. Then the magnitudes of the received symbols have the unique PDF.

Proof: Using the expression $(-1)^{s}$, where $s$ is a binary symbol, one can map the input bits 0 and 1 to 1 and -1 , respectively. Namely, $0 \rightarrow+1,1 \rightarrow-1$. Then there exists two different descriptions of received symbols; that is, $z=+1+w$ and $z=-1+w$, where $w$ is a Gaussian random variable with zero mean and variance $\sigma^{2}$, such that the PDFs of their magnitudes, denoted by $p_{1}(Z)$ and $p_{2}(Z)$ where $Z=|z|$, are given by

$$
\begin{aligned}
& p_{1}(Z)=\frac{1}{\sqrt{2 \pi} \sigma} e^{-\frac{(Z-1)^{2}}{2 \sigma^{2}}}+\frac{1}{\sqrt{2 \pi} \sigma} e^{-\frac{(-Z-1)^{2}}{2 \sigma^{2}}} \text { for } Z>0, \\
& p_{2}(Z)=\frac{1}{\sqrt{2 \pi} \sigma} e^{-\frac{(Z+1)^{2}}{2 \sigma^{2}}}+\frac{1}{\sqrt{2 \pi} \sigma} e^{-\frac{(-Z+1)^{2}}{2 \sigma^{2}}} \text { for } Z>0, \\
& p_{1}(Z)=p_{2}(Z)=\frac{1}{\sqrt{2 \pi} \sigma} e^{-\frac{1}{2 \sigma^{2}}} \text { for } Z=0 .
\end{aligned}
$$

Clearly, $p_{1}(Z)=p_{2}(Z)=p(Z)$ is valid for $Z \geq 0$. Its graphic explanation is illustrated in Fig. 1. In this figure, the blue solid line (marked with ' + ') is $p(Z)$, which is obtained by adding the red (marked with '。') and the purple (marked with ' $\square$ ') solid ones in the region $Z>0$.

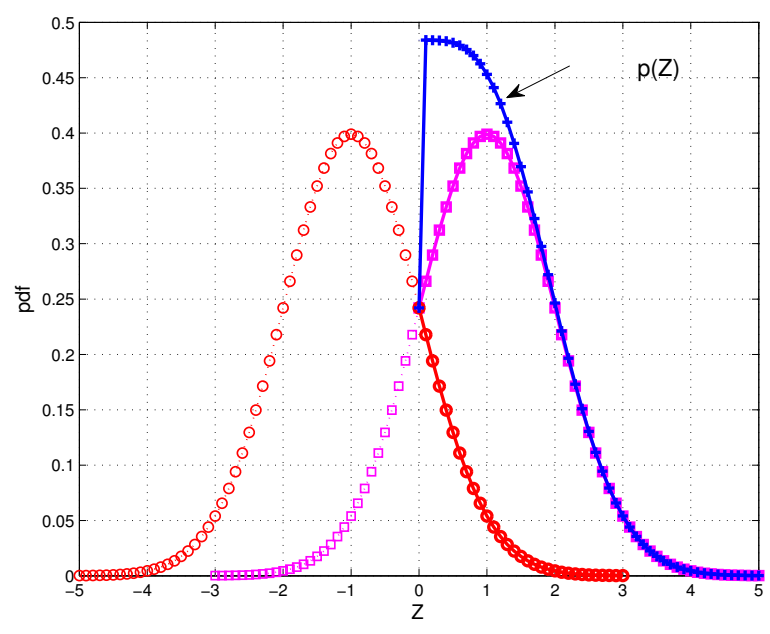

Fig. 1. The PDFs of $p_{1}(Z), p_{2}(Z)$ and $p(Z)$.

Let $A_{j}$ be the average received bit magnitude of the $j$-th coded symbol, or equivalently, $A_{j}=\frac{1}{m} \sum_{i=1}^{m}\left|z_{(j-1) * m+i}\right|$. When multiple codewords are transmitted over the burstfading AWGN channels, $\left\{A_{j}\right\}$ can be partitioned into two independent and identically distributed (i.i.d) sequences, where the coded bits are assumed to be mutually independent. The first sequence, denoted by $\boldsymbol{A}_{1}$, corresponds to pure AWGN, and the other, denoted by $\boldsymbol{A}_{\mathbf{2}}$, encounters burst fading. According to the law of large numbers, the components in $\boldsymbol{A}_{\mathbf{1}}$ approximately satisfy a Gaussian distribution. On the other hand, the distribution of the components in $\boldsymbol{A}_{\mathbf{2}}$ changes with the variation of $\sigma_{a}$. We observe that this distribution is similar to Gaussian distribution when $\sigma_{a} \leq 1.0$. The histograms of the average bit magnitude of the received symbols and the fitted 
normal density functions are illustrated in Fig. 2, which is obtained by transmitting 1000 randomly generated codewords of a $(255,223)$ RS code over pure AWGN and Rayleigh fading AWGN channels, respectively, where fading coefficients keep constant in a codeword period.

We define $E_{1}$ and $V_{1}$ to be the corresponding mean and variance of components in $\boldsymbol{A}_{1}$. Then we have

$$
\begin{aligned}
E_{1} & =E\left[\left(\frac{1}{m} \sum_{i=1}^{m}\left|r_{i}\right|\right) \mid h=1.0\right] \\
& =\frac{1}{m} \sum_{i=1}^{m} E\left[\left(\left|r_{i}\right|\right) \mid h=1.0\right] \\
& =\frac{1}{m} \cdot m \int_{0}^{+\infty} Z p(Z) d Z \\
& =\int_{0}^{0+} Z p(Z) d Z+\int_{0+}^{+\infty} Z p(Z) d Z \\
& \approx \int_{0+}^{+\infty} Z\left(\frac{1}{\sqrt{2 \pi} \sigma} e^{-\frac{(Z-1)^{2}}{2 \sigma^{2}}}+\frac{1}{\sqrt{2 \pi} \sigma} e^{-\frac{(-Z-1)^{2}}{2 \sigma^{2}}}\right) d Z \\
& =\frac{1}{2}\left(\operatorname{erfc}\left(\frac{-1}{\sqrt{2} \sigma}\right)-\operatorname{erfc}\left(\frac{1}{\sqrt{2} \sigma}\right)\right)+\frac{\sqrt{2} \sigma}{\sqrt{\pi}} e^{\frac{-1}{2 \sigma^{2}}} . \quad(14)
\end{aligned}
$$

and

$$
\begin{aligned}
V_{1}= & \operatorname{Var}\left[\left(\frac{1}{m} \sum_{i=1}^{m}\left|r_{i}\right|\right) \mid h=1.0\right] \\
= & \frac{1}{m^{2}} \cdot m \cdot \operatorname{Var}\left[\left(\left|r_{1}\right|\right) \mid h=1.0\right] \\
= & \frac{1}{m}\left(E\left[\left|r_{1}\right|^{2} \mid h=1.0\right]-E_{1}^{2}\right) \\
\approx & \left(\int_{0+}^{+\infty} Z^{2}\left(\frac{1}{\sqrt{2 \pi} \sigma} e^{-\frac{(Z-1)^{2}}{2 \sigma^{2}}}+\frac{1}{\sqrt{2 \pi} \sigma} e^{-\frac{(-Z-1)^{2}}{2 \sigma^{2}}}\right) d Z\right. \\
& \left.-E_{1}^{2}\right) \cdot \frac{1}{m} \\
= & \frac{1}{m}\left(1+\sigma^{2}-E_{1}^{2}\right) .
\end{aligned}
$$

Herein, $E[\cdot]$ and $\operatorname{Var}[\cdot]$ denote the expectation function and the variance function, respectively, and $r_{i}, i=1, \ldots, m$ denote $m$ channel observations corresponding to $m$ bits of a coded symbol. The expectation $E_{2}$ and the variance $V_{2}$ corresponding to $\boldsymbol{A}_{2}$ can be determined in a manner similar to the above equations ( $a$ is a rayleigh variable) as follows:

$$
\begin{aligned}
E_{2}= & E\left[E\left[\left(\frac{1}{m} \sum_{i=1}^{m}\left|r_{i}\right|\right) \mid h=a\right]\right] \\
\approx & \int_{0}^{+\infty}\left[\int _ { 0 + } ^ { + \infty } Z \left(\frac{1}{\sqrt{2 \pi} \sigma} e^{-\frac{(Z-a)^{2}}{2 \sigma^{2}}}+\right.\right. \\
& \left.\left.\frac{1}{\sqrt{2 \pi} \sigma} e^{-\frac{(-Z-a)^{2}}{2 \sigma^{2}}}\right) d Z\right] p(a) d a \\
= & \int_{0}^{+\infty}\left[\frac{a}{2}\left(\operatorname{erfc}\left(\frac{-a}{\sqrt{2} \sigma}\right)-\operatorname{erfc}\left(\frac{a}{\sqrt{2} \sigma}\right)\right)+\right. \\
& \left.\frac{\sqrt{2} \sigma}{\sqrt{\pi}} e^{\frac{-a^{2}}{2 \sigma^{2}}}\right] p(a) d a .
\end{aligned}
$$

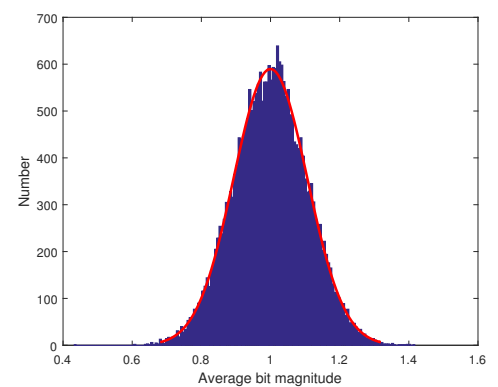

(a) The histogram of the components in $\boldsymbol{A}_{\mathbf{1}}$

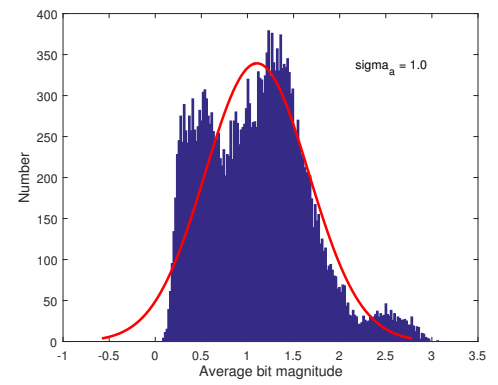

(b) The histogram of the components in $\boldsymbol{A}_{\mathbf{2}}$ with $\sigma_{a}=1.0$

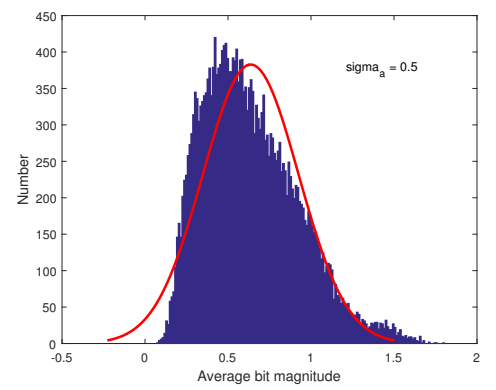

(c) The histogram of the components in $\boldsymbol{A}_{\mathbf{2}}$ with $\sigma_{a}=0.5$

Fig. 2. The histogram of the components in $\boldsymbol{A}_{\mathbf{1}}$ and $\boldsymbol{A}_{\mathbf{2}}$

and

$$
\begin{aligned}
V_{2}= & E\left[\operatorname{Var}\left[\left(\frac{1}{m} \sum_{i=1}^{m}\left|r_{i}\right|\right) \mid h=a\right]\right] \\
\approx & \int_{0}^{+\infty}\left[\frac { 1 } { m } \left(\int _ { 0 + } ^ { + \infty } Z ^ { 2 } \left(\frac{1}{\sqrt{2 \pi} \sigma} e^{-\frac{(Z-a)^{2}}{2 \sigma^{2}}}+\right.\right.\right. \\
& \left.\left.\left.\frac{1}{\sqrt{2 \pi} \sigma} e^{-\frac{(-Z-a)^{2}}{2 \sigma^{2}}}\right) d Z-E_{2}^{2}\right)\right] p(a) d a \\
= & \frac{1}{m}\left(\int _ { 0 } ^ { + \infty } \left[\int _ { 0 + } ^ { + \infty } Z ^ { 2 } \left(\frac{1}{\sqrt{2 \pi} \sigma} e^{-\frac{(Z-a)^{2}}{2 \sigma^{2}}}+\right.\right.\right. \\
& \left.\left.\left.\frac{1}{\sqrt{2 \pi} \sigma} e^{-\frac{(-Z-a)^{2}}{2 \sigma^{2}}}\right) d Z\right] p(a) d a-E_{2}^{2}\right) \\
= & \frac{1}{m}\left(\int_{0}^{+\infty}\left[a^{2}+\sigma^{2}\right] p(a) d a-E_{2}^{2}\right) .
\end{aligned}
$$

When the coded symbols are transmitted over pure AWGN channels with no fading, the errors are often randomly distributed. This implies that there is, most likely, at most one erroneous bit in each symbol. In this case, the erroronly decoder of RS codes is utilized. Contrarily, there exists 
burst errors and random errors when the signal is affected by burst fading and AWGN, and hence several bit errors are regularly occurred in a coded symbol. Those symbols containing multiple erroneous bits can be treated as erasures since they are very likely incorrect. Consequently, the RS decoder for correcting errors and erasures is employed instead. Nevertheless, it is very difficult to determine the exact starting and ending points of burst fading in a codeword period. In this paper, we arrive at a scheme without estimating the starting point and the length of burst fading to decode RS codes over the burst Rayleigh fading channels. The complete decoding algorithm, called Algorithm 1, is summarized below:

\section{Algorithm 1:}

1) Initially, compute $E_{1}, E_{2}, V_{1}, V_{2}$, and $A_{j}$, where $1 \leq$ $j \leq n$. The symbol $E_{2}$ can be considered as a special reliability of a coded symbol located in the burst-fading region since it is the mean of the average received bit magnitude of a coded symbol. Based on the idea given in [27], the corresponding error probability has the form

$$
T_{s}=\frac{1}{1+e^{\frac{2 E_{2}}{\sigma^{2}}}} .
$$

This parameter will be hereinafter used as a threshold. If the bit-error probability of a coded bit exceeds this threshold, then it is more likely erroneous.

2) Given a coded symbol $y_{j}$, if its average bit magnitude $A_{j}<A^{*}$, then this symbol can be supposed to be located in the burst-fading region. Here, the parameter $A^{*}$ is the intersection of two PDF functions $f_{1}(u)=$ $\frac{1}{\sqrt{2 \pi V_{1}}} e^{-\frac{\left(u-E_{1}\right)^{2}}{2 V_{1}}}$ and $f_{2}(u)=\frac{1}{\sqrt{2 \pi V_{2}}} e^{-\frac{\left(u-E_{2}\right)^{2}}{2 V_{2}}}$ and can be obtained according to Eq. (19).

3) The channel model in the burst fading region is $z=$ $\pm a+w$, where $z$ and $w$ are the channel output and a AWGN noise symbol, respectively. Assume that ' 0 ' and ' 1 ' are uniformly distributed. The average bit-error probability corresponding to the burst fading region can be written explicitly as follows:

$$
\begin{aligned}
p_{B F}= & E\left[p_{a} \mid a\right] \\
= & \int_{0}^{+\infty}\left[\frac{1}{2} \int_{-\infty}^{0} \frac{1}{\sqrt{2 \pi} \sigma} e^{\frac{-(z-a)^{2}}{2 \sigma^{2}}} d z+\right. \\
& \left.\frac{1}{2} \int_{0}^{+\infty} \frac{1}{\sqrt{2 \pi} \sigma} e^{\frac{-(z+a)^{2}}{2 \sigma^{2}}} d z\right] p(a) d a \\
= & \int_{0}^{+\infty} \int_{-\infty}^{0} \frac{1}{\sqrt{2 \pi} \sigma} e^{\frac{-(z-a)^{2}}{2 \sigma^{2}}} \cdot p(a) d z d a .
\end{aligned}
$$

Herein, $p_{a}$ denotes the average bit-error probability when the fading coefficient $a$ is given. Then, $L=$ $\left\lceil p_{B F} \cdot m\right\rceil$ is the expectation number of erroneous bits in a coded symbol, where $\lceil x\rceil$ denotes the least integer greater than or equal to $x$. To avoid $L=1$ in some cases, $L=\max \left(\left\lceil p_{B F} \cdot m\right\rceil, 2\right)$ is chosen instead.

4) Proceed the received coded symbol $y_{j}$ for $j=1, \ldots, n$ satisfying $A_{j}<A^{*}$, and flag it as an erasure if the number of bits in a coded symbol whose bit-error probabilities satisfying the inequality $P_{j, i} \geq T_{s}$ is larger than or equal to $L^{1}$, where

$$
P_{j, i}=\frac{1}{1+e^{\frac{2 \mid z(j-1) * m+i}{\sigma^{2}}}} \text { for } i=1, \ldots, m .
$$

5) Conduct EED according to the erasure locations determined in Step 4. If no erasures are marked, then run the BM decoder.

Remarks: When we simulate Algorithm 1, we find that $\left\lceil p_{B F} \cdot m\right\rceil$ is typically equal to 1 or 2 in the tested SNR regimes. Therefore, Step 3 of Algorithm 1 can be omitted by fixing $L$ to 2 . Moreover, it can readily be understood that the choice of the bit-error probability threshold in Step 1 of Algorithm 1 is extremely crucial for the error-rate performance of this algorithm.

In Step 2 of the above algorithm, the probability that a received symbol located in the pure AWGN region is mistaken as being located in the burst fading region is given by the formula

$$
\hat{p}=\int_{0}^{A^{*}} \frac{1}{\sqrt{2 \pi V_{1}}} e^{\frac{-\left(Z-E_{1}\right)^{2}}{2 V_{1}}} d Z .
$$

One observes from Fig. 3 that this probability is high for $\sigma_{a} \geq$ 0.5, which deteriorates the performance of Algorithm 1 since several correct symbols located in the pure AWGN region are marked as erasures.

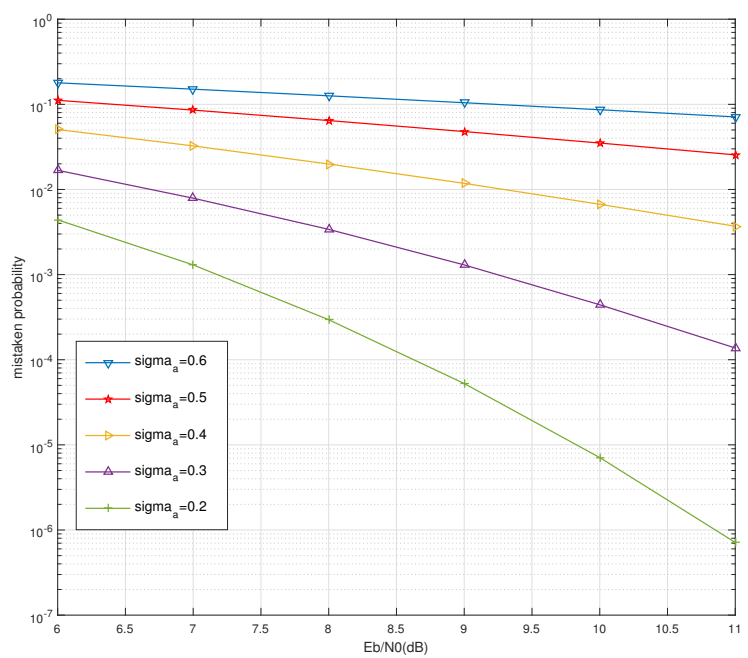

Fig. 3. The probability that a received symbol located in the pure AWGN region is mistaken as being located in the burst fading region for different $\sigma_{a}$.

If the starting and ending points of the burst fading are known, then $A^{*}$ does not need to be determined. Consequently, an improved version of Algorithm 1, called Algorithm 2, is thus obtained as follows:

Algorithm 2:

1) Compute $A_{j}$ for $j=1,2, \ldots, n$ and $T_{s}$.

2) Determine $L$ in a manner similar to Step 3 of Algorithm 1 (or simply set $L$ to 2 ).

\footnotetext{
${ }^{1}$ Note that the expression $1 /\left(1+e^{2 x / \sigma^{2}}\right), x \geq 0$, is a monotonically decreasing function. Thus, we only need to compare $\left|z_{(j-1) * m+i}\right|$ with $E_{2}$ when programming Algorithm 1.
} 


$$
A^{*}=\frac{\left(E_{2} V_{1}-E_{1} V_{2}+V_{1} V_{2} \sqrt{\left(\left(E_{1}-E_{2}\right)^{2}-2 V_{1} \log \left(\sqrt{\frac{V_{2}}{V_{1}}}\right)+2 V_{2} \log \left(\sqrt{\frac{V_{2}}{V_{1}}}\right)\right) /\left(V_{1} V_{2}\right)}\right)}{V_{1}-V_{2}} .
$$

3) Proceed the received symbols located in the burst fading region $y_{j}, \forall j \in\left[f_{s}, f_{e}\right]$, where $f_{s}$ and $f_{e}$ denote the starting point and ending points of burst fading, respectively, and flag a received symbol as an erasure if the number of bits in this symbol whose bit-error probabilities satisfying the inequality $P_{j, i} \geq T_{s}$ is larger than or equal to $L$.

4) Conduct EED in accordance with the erasure locations determined in Step 3. If no erasures are flagged, then run the BM decoder.

\section{B. Performance Analysis Of New Decoding Algorithms}

We first derive a formula to estimate the performance of Algorithm 2 in which the locations of the burst fading are known in advance. Assume that a $(n, k) \mathrm{RS}$ code over $G F\left(2^{m}\right)$ is transmitted over the burst Rayleigh fading channels with BPSK modulation and Algorithm 2 is used to decode this code. Without loss of generality, all-zero codewords are always transmitted. The bit-error probability of each coded bit in the pure AWGN region is given by

$$
p_{A}=\int_{-\infty}^{0} \frac{1}{\sqrt{2 \pi} \sigma} e^{\frac{-(z-1)^{2}}{2 \sigma^{2}}} d z
$$

We assume that the coded bits are mutually independent, then the probability that a coded symbol in the pure AWGN region is an error can be written as

$$
P_{E 1}=1-\left(1-p_{A}\right)^{m}
$$

In the burst-fading region, an incorrect coded symbol is handled as an erasure with high probability. It is interesting to note that a correct received symbol might be mistaken as an erasure by the decoder based on Algorithm 2. Upon inspection of the proposed algorithm, one knows that the $j$-th coded symbol is an erasure if and only if there exist at least $L$ bits in that symbol whose corresponding channel observations have magnitudes less than or equal to $E_{2}$. Let $r$ be the channel observation of any coded bit located in the burst fading region, then the probability that a coded symbol in the burst-fading region will be marked as an erasure can be formulated as

$$
P_{\text {era }}=\sum_{u=L}^{m} C_{m}^{u}\left[\operatorname{Pr}\left\{|r| \leq E_{2}\right\}\right]^{u}\left[\operatorname{Pr}\left\{|r|>E_{2}\right\}\right]^{m-u}
$$

Herein,

$$
\begin{aligned}
\operatorname{Pr} & \left\{|r|>E_{2}\right\} \\
= & \operatorname{Pr}\left\{r>E_{2}\right\}+\operatorname{Pr}\left\{r<-E_{2}\right\} \\
= & \int_{E_{2}}^{+\infty} f(r) d r+\int_{-\infty}^{-E_{2}} f(r) d r \\
= & \int_{E_{2}}^{+\infty} \frac{1}{\sqrt{2 \pi\left(\operatorname{Var}[a]+\sigma^{2}\right)}} e^{\frac{-(r-E[a])^{2}}{2\left(\operatorname{Var}[a]+\sigma^{2}\right)}} d r \\
& +\int_{-\infty}^{-E_{2}} \frac{1}{\sqrt{2 \pi\left(\operatorname{Var}[a]+\sigma^{2}\right)}} e^{\frac{-(r-E[a])^{2}}{2\left(\operatorname{Var}[a]+\sigma^{2}\right)}} d r \\
= & \frac{1}{2} \operatorname{erfc}\left(\frac{E_{2}-E[a]}{\sqrt{2\left(\operatorname{Var}[a]+\sigma^{2}\right)}}\right)+ \\
& \frac{1}{2} \operatorname{erfc}\left(\frac{E_{2}+E[a]}{\sqrt{2\left(\operatorname{Var}[a]+\sigma^{2}\right)}}\right),
\end{aligned}
$$

where

$$
\begin{aligned}
E[a] & =\int_{0}^{+\infty} a p(a) d a \\
& =\sqrt{\frac{\pi}{2}} \sigma_{a} . \\
\operatorname{Var}[a] & =E\left[a^{2}\right]-(E[a])^{2}=\frac{4-\pi}{2} \sigma_{a}^{2} .
\end{aligned}
$$

Here, the third equality in Eq. (26) uses the fact that $r$ is approximately Gaussian distributed in the referred channels. Then we obtain $P_{\text {era }}$ by substituting Eq. (26) into Eq. (25), where $\operatorname{Pr}\left\{|r| \leq E_{2}\right\}=1-\operatorname{Pr}\left\{|r|>E_{2}\right\}$. Accordingly, the probability that a wrong received symbol in the burst fading region is not marked as an erasure is given by

$$
P_{E 2}=\left(1-\left(1-p_{B F}\right)^{m}\right)\left(1-P_{e r a}\right) .
$$

The probability of successful decoding by utilizing Algorithm 2 can be clearly expressed as

$$
P_{s}=\operatorname{Pr}\left\{2 N_{e}+N_{\text {era }} \leq d-1\right\},
$$

where $N_{e}$ and $N_{\text {era }}$ denote the number of errors and erasures, respectively. Let $N_{e 1}$ and $N_{e 2}$ be the number of errors in the pure AWGN and burst-fading AWGN regions, respectively; that is, $N_{e}=N_{e 1}+N_{e 2}$. Then we have

$$
\begin{array}{lc}
P_{s} & \sum_{u, \beta, \gamma, \text { s.t. } 2 u+2 \gamma+\beta \leq d-1} \operatorname{Pr}\left\{N_{e 1}=u, N_{\text {era }}=\beta, N_{e 2}=\gamma\right\} \\
= & \sum_{u, \beta, \gamma, \text { s.t. } 2 u+2 \gamma+\beta \leq d-1} \operatorname{Pr}\left\{N_{e 1}=u\right\} \operatorname{Pr}\left\{N_{\text {er } a}=\beta, N_{e 2}=\gamma\right\} \\
=\sum_{u=0}^{\left\lfloor\frac{d-1}{2}\right\rfloor} \sum_{\beta=0}^{d-1} \sum_{\gamma=0}^{\left\lfloor\frac{d-1}{2}\right\rfloor}\left(\operatorname{Pr}\left\{N_{e 1}=u\right\} \operatorname{Pr}\left\{N_{\text {era }}=\beta, N_{e 2}=\gamma\right\}\right. \\
\cdot \Pi(2 u+2 \gamma+\beta \leq d-1)) .
\end{array}
$$


Here, $\Pi(\cdot)$ is the indicator function and satisfies

$$
\Pi(x)= \begin{cases}1 & x \text { is ture } \\ 0 & \text { otherwise }\end{cases}
$$

The second equality in (31) is valid because $N_{e 1}$ is independent with $N_{e 2}$ and $N_{e r a}$. Note that $N_{e 2}$ and $N_{e r a}$ are mutually dependent since both of which correspond to burstfading. To avoid the case ' $N_{e r a}+N_{e 2}>\tau$ ', where $\tau=\left\lceil\frac{L_{b}}{m}\right\rceil$ represents the number of coded symbols in the burst fading region, we state that $C_{i}^{j}=0$ if $i<j$ when the probability $\operatorname{Pr}\left\{N_{\text {era }}=\beta, N_{e 2}=\gamma\right\}$ is determined as follows:

$$
\begin{aligned}
\operatorname{Pr}\left\{N_{e r a}=\beta, N_{e 2}=\gamma\right\}= & C_{\tau}^{\beta}\left(P_{\text {era }}\right)^{\beta} C_{\tau-\beta}^{\gamma}\left(P_{E 2}\right)^{\gamma} . \\
& \left(1-P_{\text {era }}-P_{E 2}\right)^{\tau-\beta-\gamma} .
\end{aligned}
$$

Additionally, it follows from Eqs. (23) and (24) that

$$
\operatorname{Pr}\left\{N_{e 1}=u\right\}=C_{n-\tau}^{u}\left(P_{E 1}\right)^{u}\left(1-P_{E 1}\right)^{n-\tau-u} .
$$

Similar to the analysis given in [24], combining Eqs. (31), (33), and (34) yields $P_{s}$ as given in (31) and hence the frame error rate (FER) is obtained immediately from the following equation:

$$
\begin{aligned}
& \text { FER } \\
& \begin{aligned}
=\quad & 1-P_{s} \\
= & 1-\sum_{u=0}^{\left\lfloor\frac{d-1}{2}\right\rfloor} \sum_{\beta=0}^{d-1} \sum_{\gamma=0}^{\left\lfloor\frac{d-1}{2}\right\rfloor}\left[C_{n-\tau}^{u}\left(P_{E 1}\right)^{u}\left(1-P_{E 1}\right)^{n-\tau-u} .\right. \\
& C_{\tau}^{\beta}\left(P_{\text {era }}\right)^{\beta} C_{\tau-\beta}^{\gamma}\left(P_{E 2}\right)^{\gamma}\left(1-P_{\text {era }}-P_{E 2}\right)^{\tau-\beta-\gamma} . \\
& \Pi(2 u+2 \gamma+\beta \leq d-1)] .
\end{aligned}
\end{aligned}
$$

When Algorithm 1 is utilized, the probability that a received symbol in the burst-fading region is marked as an erasure is approximately computed by

$$
P_{e r a, 2}^{\prime}=\int_{0}^{A *} \frac{1}{\sqrt{2 \pi V_{2}}} e^{\frac{-\left(Z-E_{2}\right)^{2}}{2 V_{2}}} d Z \cdot P_{\text {er } a} .
$$

Correspondingly, the probability that an incorrect received symbol in the burst fading region fails to be marked as an erasure is determined by

$$
P_{E 2}^{\prime}=\left(1-\left(1-p_{B F}\right)^{m}\right)\left(1-P_{e r a, 2}^{\prime}\right) .
$$

Moreover, some received symbols in the pure AWGN region are mistaken as being located in the burst fading region, and they might also be flagged as erasures. Similarly, the corresponding erasure probability, denoted by $P_{e r a, 1}^{\prime}$, is given by

$$
\begin{aligned}
P_{\text {era }, 1}^{\prime}= & \int_{0}^{A *} \frac{1}{\sqrt{2 \pi V_{1}}} e^{\frac{-\left(Z-E_{1}\right)^{2}}{2 V_{1}}} d Z . \\
& {\left[\sum_{u=L}^{m} C_{m}^{u}\left[\operatorname{Pr}\left\{\left|r^{\prime}\right| \leq E_{2}\right\}\right]^{u}\left[\operatorname{Pr}\left\{\left|r^{\prime}\right|>E_{2}\right\}\right]^{m-u}\right], }
\end{aligned}
$$

where $r^{\prime}$ is the channel observation of a coded bit located in the pure AWGN region and the probability $\operatorname{Pr}\left\{\left|r^{\prime}\right|>E_{2}\right\}$ is computed by

$$
\begin{aligned}
\operatorname{Pr}\left\{\left|r^{\prime}\right|>E_{2}\right\}= & \operatorname{Pr}\left\{r^{\prime}>E_{2}\right\}+\operatorname{Pr}\left\{r^{\prime}<-E_{2}\right\} \\
= & \int_{E_{2}}^{+\infty} f\left(r^{\prime}\right) d r^{\prime}+\int_{-\infty}^{-E_{2}} f\left(r^{\prime}\right) d r^{\prime} \\
= & \int_{E_{2}}^{+\infty} \frac{1}{\sqrt{2 \pi \sigma^{2}}} e^{\frac{-\left(r^{\prime}-1\right)^{2}}{2 \sigma^{2}}} d r^{\prime} \\
& +\int_{-\infty}^{-E_{2}} \frac{1}{\sqrt{2 \pi \sigma^{2}}} e^{\frac{-\left(r^{\prime}-1\right)^{2}}{2 \sigma^{2}}} d r^{\prime} \\
= & \frac{1}{2} \operatorname{erfc}\left(\frac{E_{2}-1}{\sqrt{2 \sigma^{2}}}\right)+\frac{1}{2} \operatorname{erfc}\left(\frac{E_{2}+1}{\sqrt{2 \sigma^{2}}}\right) .
\end{aligned}
$$

Accordingly, the probability that an incorrect received symbol in the pure AWGN region is not marked as an erasure is given by

$$
P_{E 1}^{\prime}=\left(1-\left(1-p_{A}\right)^{m}\right)\left(1-P_{e r a, 1}^{\prime}\right) .
$$

Let $N_{e r a, 2}^{\prime}$ and $N_{e 2}^{\prime}\left(N_{e r a, 1}^{\prime}\right.$ and $\left.N_{e 1}^{\prime}\right)$ be the number of erasures and errors in the burst fading (pure AWGN) region, respectively. Similar to the above analysis, the probability of successful decoding by utilizing Algorithm 1 is given by

$$
\begin{aligned}
P_{s}^{\prime}= & \sum_{\substack{v, u, \beta, \gamma, s . t . \\
2 u+v+2 \gamma+\beta \leq d-1}} \operatorname{Pr}\left\{\begin{array}{l}
N_{e r a, 1}^{\prime}=v, N_{e 1}^{\prime}=u \\
N_{e r a, 2}^{\prime}=\beta, N_{e 2}^{\prime}=\gamma
\end{array}\right\} \\
= & \sum_{\substack{v, u, \beta, \gamma, s . t . \\
2 u+v+2 \gamma+\beta \leq d-1}} \operatorname{Pr}\left\{N_{e r a, 1}^{\prime}=v, N_{e 1}^{\prime}=u\right\} . \\
& \operatorname{Pr}\left\{N_{e r a, 2}^{\prime}=\beta, N_{e 2}^{\prime}=\gamma\right\} .
\end{aligned}
$$

According to the derivation of Eq. (31), the corresponding FER of Algorithm 1, denoted by FER', is directly obtained by combining Eqs. (36), (40), and (41) as follows:

$$
\begin{array}{ll}
\text { FER }^{\prime} & \\
= & 1-P_{s}^{\prime} \\
= & 1-\sum_{v=0}^{d-1} \sum_{u=0}^{\left\lfloor\frac{d-1}{2}\right\rfloor} \sum_{\beta=0}^{d-1} \sum_{\gamma=0}^{\left\lfloor\frac{d-1}{2}\right\rfloor}\left[C_{n-\tau}^{v}\left(P_{e r a, 1}^{\prime}\right)^{v} C_{n-\tau-v}^{u} \cdot\right. \\
& \left(P_{E 1}^{\prime}\right)^{u}\left(1-P_{e r a, 1}^{\prime}-P_{E 1}^{\prime}\right)^{n-\tau-v-u} \cdot C_{\tau}^{\beta}\left(P_{e r a, 2}^{\prime}\right)^{\beta} . \\
& C_{\tau-\beta}^{\gamma}\left(P_{E 2}^{\prime}\right)^{\gamma}\left(1-P_{e r a, 2}^{\prime}-P_{E 2}^{\prime}\right)^{\tau-\beta-\gamma} . \\
& \Pi(2 u+v+2 \gamma+\beta \leq d-1)] .
\end{array}
$$

Proposition 2: Assume that an $(n, k)$ RS code over $G F\left(2^{m}\right)$ is transmitted over burst Rayleigh fading channels and the BM algorithm is utilized to decode this code. Then the FER can be explicitly expressed as

$$
\begin{aligned}
& \mathrm{FER}_{B M} \\
& \begin{aligned}
=\quad & 1-\sum_{\gamma=0}^{\left\lfloor\frac{d-1}{2}\right\rfloor} \sum_{u=0}^{\gamma}\left[C_{n-\tau}^{u}\left(P_{E 1}\right)^{u}\left(1-P_{E 1}\right)^{n-\tau-u} .\right. \\
& C_{\tau}^{\gamma-u}\left(1-\left(1-p_{B F}\right)^{m}\right)^{\gamma-u} . \\
& \left.\left(\left(1-p_{B F}\right)^{m}\right)^{\tau-(\gamma-u)}\right]
\end{aligned}
\end{aligned}
$$

Proof: For simplicity, the same notations mentioned above are used. It is well-known that the BM algorithm can 
correct up to $\left\lfloor\frac{d-1}{2}\right\rfloor$ errors. Thus, the probability of successful decoding is given by

$$
\begin{aligned}
P_{s}^{\prime \prime}= & \operatorname{Pr}\left\{N_{e 1}+N_{e 2} \leq\left\lfloor\frac{d-1}{2}\right\rfloor\right\} \\
= & \sum_{\gamma=0}^{\left\lfloor\frac{d-1}{2}\right\rfloor} \operatorname{Pr}\left\{N_{e 1}+N_{e 2}=\gamma\right\} \\
= & \sum_{\gamma=0}^{\left\lfloor\frac{d-1}{2}\right\rfloor} \sum_{u=0}^{\gamma} \operatorname{Pr}\left\{N_{e 1}=u\right\} \cdot \operatorname{Pr}\left\{N_{e 2}=\gamma-u\right\} \\
= & \sum_{\gamma=0}^{\left\lfloor\frac{d-1}{2}\right\rfloor} \sum_{u=0}^{\gamma}\left[C_{n-\tau}^{u}\left(P_{E 1}\right)^{u}\left(1-P_{E 1}\right)^{n-\tau-u} C_{\tau}^{\gamma-u} .\right. \\
& \left.\left(1-\left(1-p_{B F}\right)^{m}\right)^{\gamma-u}\left(\left(1-p_{B F}\right)^{m}\right)^{\tau-(\gamma-u)}\right] .
\end{aligned}
$$

The $\mathrm{FER}_{B M}$ in (43) immediately follows from Eq. (44). The proof of Proposition 2 is thus complete.

\section{Simulation RESUlts}

In this section, simulations of the proposed and the existing decoding algorithms for RS codes in the burst Rayleigh fading channels with BPSK modulation are conducted using the C programming language. Typically, we choose the BM algorithm, Wu's algorithm [22] and the IEED algorithm given in [18] as counterparts. Hereafter, we let ' $\operatorname{IEED}(\lambda, \eta)$ ' denote the IEED algorithm with the parameters $\lambda$ and $\eta$. In reality, we run Algorithm 3 given in [22] when conducting Wu's algorithm, in which a supposed maximum number of random errors $\delta$ needs to be preset. When $\delta$ is a small constant, Wu's algorithm requires $O(v n)$ complexity, whereas such a complexity will gradually increase up to $O\left(v^{2} n\right)$ with the increasement of $\delta$. Furthermore, increasing $\delta$ may result in a performance loss in our applications since the maximum length of bursts that can be determined will shorten. Thus, an appropriate $\delta$ needs to be chosen in different scenarios. Consider three different systematic RS codes with the following parameters:

Code $C_{1}: n=255, k=223$;

Code $C_{2}: n=1023, k=959$;

Code $C_{3}: n=528, k=514$.

In order to demonstrate the performance of the proposed algorithms, cases of $\sigma_{a}=0.2, \sigma_{a}=0.3$ and $\sigma_{a}=0.4$ are tested with four different fading configurations: 200-bit, 120-bit, 400-bit and 100-bit consecutive fading segments. For comparison purposes, with the aid of Eqs. (35), (42) and (43), the FER performance of the new algorithms and the $\mathrm{BM}$ algorithm are mathematically estimated. All of simulation curves are obtained when collecting 100 codeword errors unless otherwise specified. The performance of the abovementioned decoding methods are illustrated by the following three examples:

\section{A. Example 1}

The comparisons of FER performance for the four different decoding algorithms with code $C_{1}$ and 200-bit consecutive burst fading under two fading parameters are demonstrated in Figs. 4-5. It can be seen from Fig. 4 that Algorithm 1 achieves a substantial gain over the classic BM algorithm. Meanwhile, this algorithm is approximately $0.4 \mathrm{~dB}$ better than Wu's algorithm $(\delta=1)$ but is inferior to the IEED $(\lambda=26$, $\eta=12$ ) algorithm by about $0.6 \mathrm{~dB}$, respectively, at FER of $10^{-4}$. In order to compare Algorithm 1 with Wu's algorithm more deeply, the performance of the latter in the ideal situation is also simulated by assuming the number of random errors is known in advance. One observes from Fig. 4 that the ideal Wu's algorithm performs about $0.45 \mathrm{~dB}$ better than Algorithm 1. Furthermore, Algorithm 2 slightly outperforms the IEED algorithm. It is worth noting that the IEED algorithm has a very high computational complexity. Therefore, only 20 frame errors are collected when declaring a FER of this algorithm in the high SNR regimes. Nevertheless, Algorithm 1 performs well despite without the knowledge of the fading locations and requires a low complexity. We observe that the gap between the performance estimated from Eq. (42) and the simulation curve for Algorithm 1 is approximately $0.5 \mathrm{~dB}$. Whereas, Eq. (43) cannot predict the performance of the BM algorithm correctly since the fading coefficient $a$ takes values in a relatively large interval. It is of interest that the performance analysis of Algorithm 2 is highly consistent with the simulation results, in which the burst fading locations are assumed to be known.

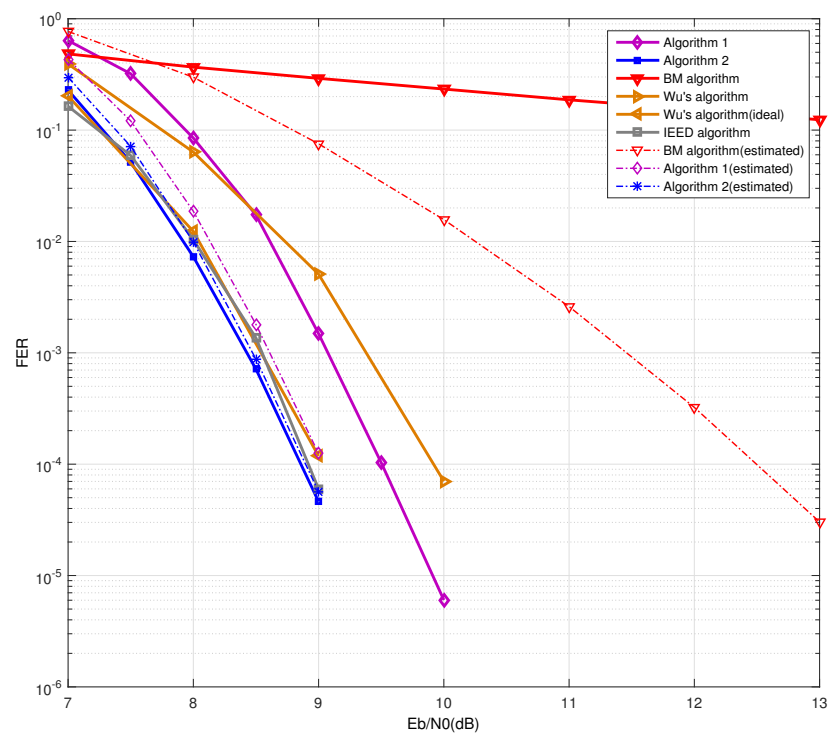

Fig. 4. FER performance of code $C_{1}$ over burst Rayleigh fading channels with $\sigma_{a}=0.4$.

In Fig. 5, the performance of five decoding algorithms when $\sigma_{a}=0.3$ is compared. Observations illustrate that Algorithm 1 far outperforms the BM algorithm at the whole tested SNR regimes. In this case, Algorithm 2 has only a gain of about $0.1 \mathrm{~dB}$ over Algorithm 1 despite the known burst locations. Algorithm 1 is $1.0 \mathrm{~dB}$ better than Wu's algorithm $(\delta=1)$ at FER of $2 \times 10^{-4}$ and even outperforms Wu's algorithm with the ideal case. In this scenario, the IEED $(\lambda=25, \eta=10)$ algorithm is inferior to our proposed algorithms. Moreover, Eq. (35) estimates the performance of Algorithm 2 very accurately for moderate to high SNR, and the gap between 
the simulated and the estimated curves for Algorithm 1 is merely $0.1 \mathrm{~dB}$.

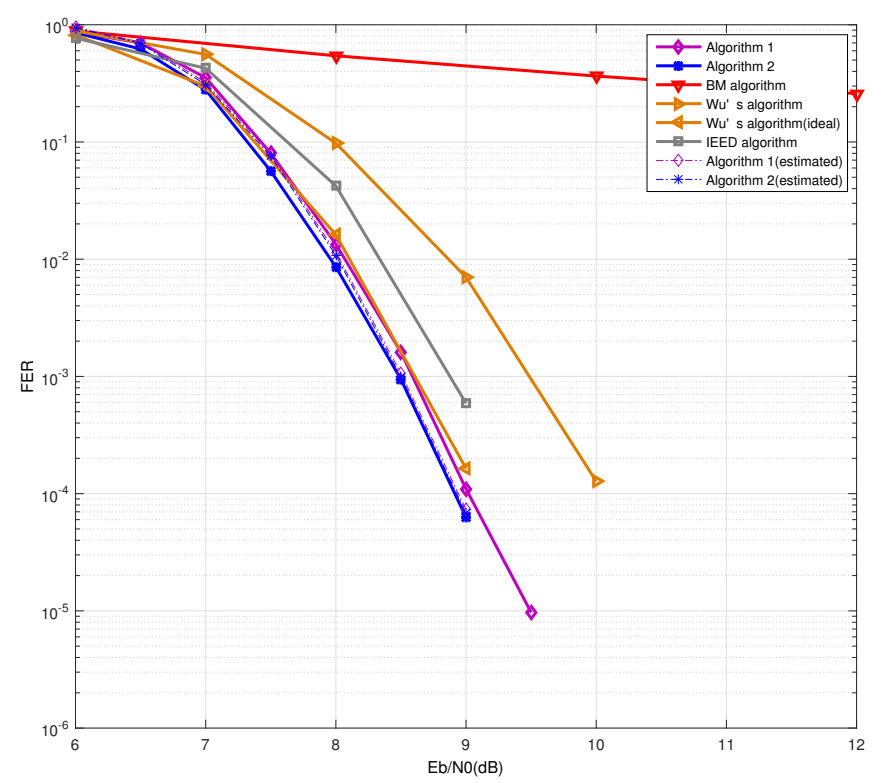

Fig. 5. FER simulations of code $C_{1}$ over burst Rayleigh fading channels with $\sigma_{a}=0.3$.

\section{B. Example 2}

In this example, the above five decoding algorithms are compared in Fig. 6 when the code $C_{1}$ and 120-bit consecutive fading are considered. The fading parameters $\sigma_{a}$ is equal to 0.2 . It can be inferred from Fig. 6 that Algorithm 1 is approximately $2.5 \mathrm{~dB}$ better than the BM algorithm at FER of $2 \times 10^{-4}$. The performance difference between Algorithms 1 and 2 is less than $0.1 \mathrm{~dB}$ although the former has no information on burst-fading locations. Moreover, Algorithm 1 provides almost $0.3 \mathrm{~dB}$ gain over Wu's algorithm $(\delta=6)$ at FER of $10^{-4}$ but performs worse than the IEED $(\lambda=16, \eta=10)$ algorithm. In this example, Eqs. (35) and (42) still anticipate the FER performance relatively accurately. Nevertheless, Eq. (43) cannot estimate the performance correctly.

As shown in Fig. 7, when a longer code, namely $C_{2}$, is utilized and 400-bit burst fading is encountered, the BM decoder doesn't work. Simultaneously, Algorithm 1 has the almost completely same performance as Algorithm 2 in the whole tested SNR regimes. This means that Steps 2 and 4 in Algorithm 1 jointly avoid treating a coded symbol that encounters no fading as an erasure with a probability close to 1.0. Furthermore, our algorithms have approximately $0.3 \mathrm{~dB}$ and $0.2 \mathrm{~dB}$ gains when compared with Wu's algorithm $(\delta=9)$ and the IEED $(\lambda=41, \eta=10)$ algorithm, respectively. In this example, Eqs. (35) and (42) predict the FER performance very accurately.

\section{Example 3}

Fig. 8 demonstrates the error-correction performance of code $C_{3}$ when 100-bit burst fading is encountered with $\sigma_{a}=$ 0.4 and $\sigma_{a}=0.2$. It is actually a shorten version of the (1023,

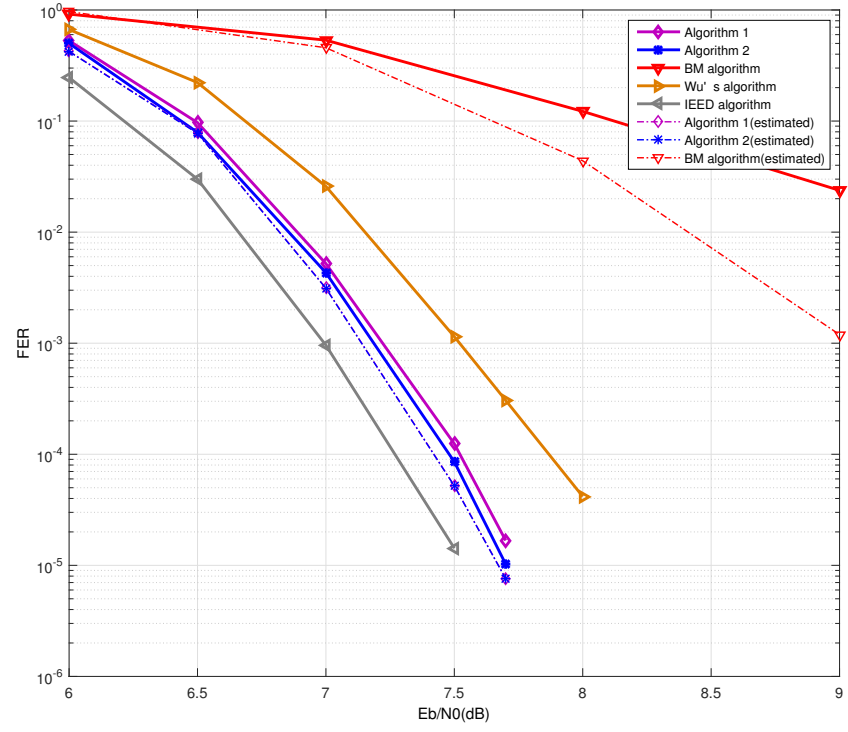

Fig. 6. FER simulations of code $C_{1}$ over burst Rayleigh fading channels with $\sigma_{a}=0.2$.

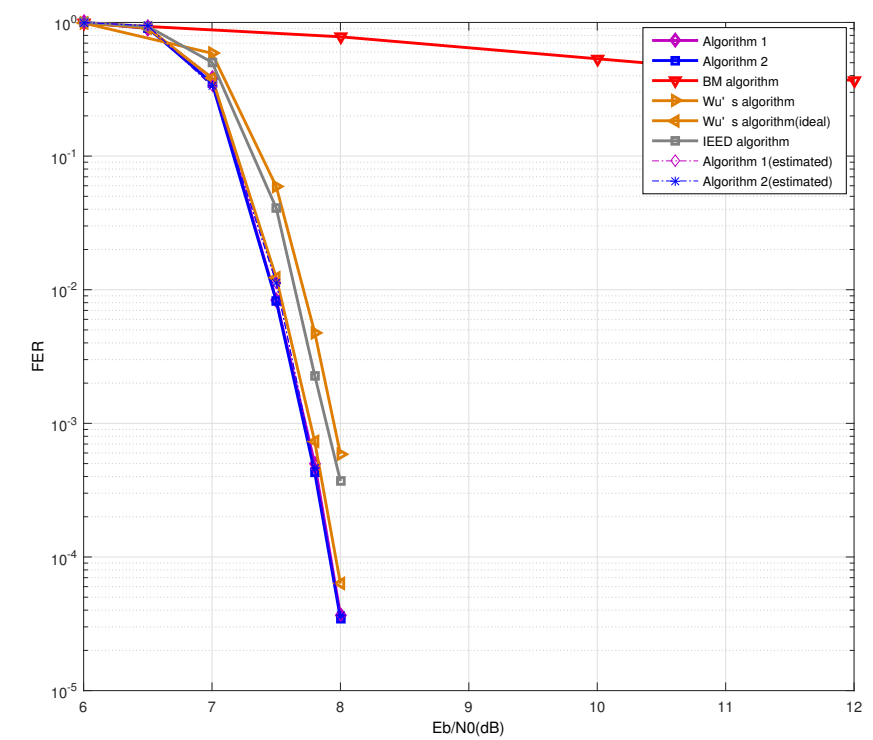

Fig. 7. FER simulations of code $C_{2}$ over burst Rayleigh fading channels with $\sigma_{a}=0.2$.

1009) RS code and has been adopted for $100 \mathrm{~Gb} / \mathrm{s}$ copper backplane transmission [30]. One observes from this figure that Algorithm 1 performs evidently worse than the IEED $(\lambda=11, \eta=10)$ algorithm when $\sigma_{a}=0.4$. In this case, Algorithm 2 performs nearly as well as the IEED algorithm. When $\sigma_{a}$ is reduced down to 0.2, Algorithm 1 exhibits a very good performance despite unknown fading locations. The gap between Algorithms 1 and 2 is merely about $0.2 \mathrm{~dB}$. As can be seen from this figure, Algorithm 1 outperforms the IEED algorithm at low-to-moderate SNR while performs a little worse than the latter in the high SNR regimes. In this example, Wu's algorithm $(\delta=1)$ exhibits evident error floors since the error correcting capability of the code $C_{3}$ is too low to correct such a long burst. In contrast, no error floor appears 
at FER $\geq 3 \times 10^{-6}$ when considering 80 -bit burst fading.

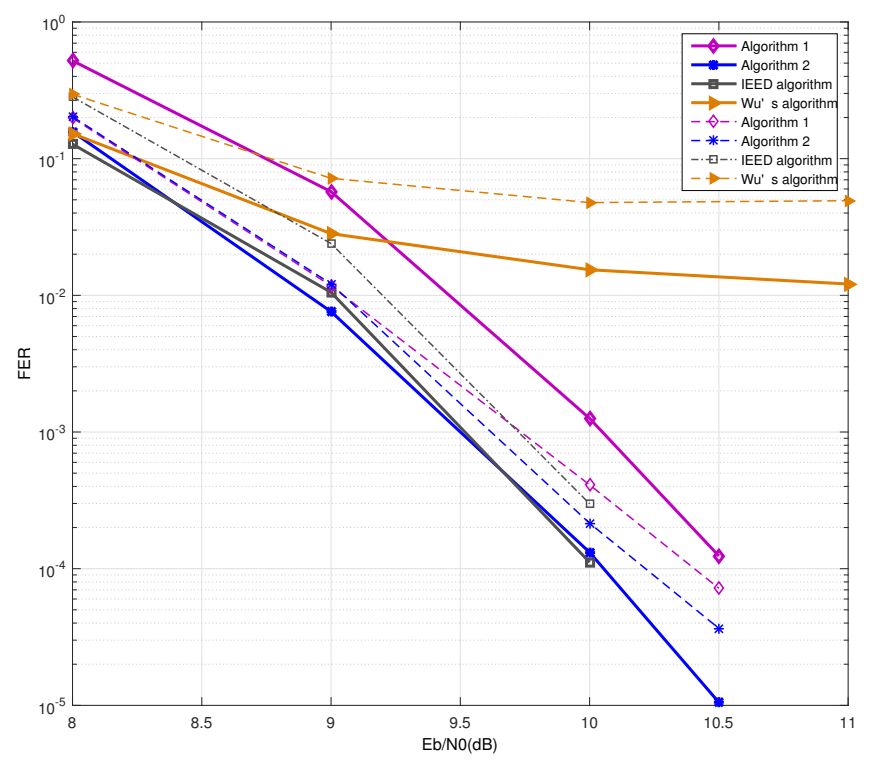

Fig. 8. FER simulation results of code $C_{3}$ when encountering 100-bit burstfading for $\sigma_{a}=0.4$ (solid lines) and $\sigma_{a}=0.2$ (dashed lines).

\section{Complexity Analysis}

In Algorithm 1, Steps 1, 2 and 3 require low calculation amounts. Hence, we mainly consider steps 4 and 5 when measuring the computational complexity of Algorithm 1. Step 4 needs $m \times n$ real-number comparisons while Step 5 has complexity $O(v n)$. As a consequence, the algorithmic complexity of Algorithms 1 and 2 is of the same order as Wu's algorithm (with a small constant $\delta$ ), $O(v n)$. In comparison, the IEED algorithm conducts $2^{\eta}$ EED, and hence has the worst-case complexity of $O\left(2^{\eta} \mathrm{vn}\right)$. It should be noted that some steps of the EED needs to be conducted $n$ times in Wu's algorithm when computing the error locator polynomial corresponding to up to $\delta$ random errors. Consequently, Algorithm 1 certainly runs faster than Wu's algorithm even if their complexities are of the same order.

For a more detailed comparison, we also test the average decoding time of Algorithm 1, Wu's algorithm $(\delta=1)$ and the IEED $(\lambda=26, \eta=12)$ algorithm on a $4.20 \mathrm{GHz}$ Intel Core i7 Processor by taking the code $C_{1}$ as an example with 200-bit burst fading and $\sigma_{a}=0.4$. As shown in Table I, Algorithm 1 averagely performs 20.6 times and 1568.8 times faster than Wu's algorithm and the IEED algorithm, respectively. Such a substantial improvement on decoding speed is reasonable since Wu's algorithm conducts some steps of the EED multiple times and the IEED algorithm runs the whole EED thousands of times, whereas Algorithm 1 only run EED once.

\section{E. Summary}

In the simulation tests mentioned above, different RS code rates, lengths, as well as various burst-fading configurations
TABLE I

COMPARISON OF AVERAGE CPU RUNTIME OF DECODING A CODEWORD (MS) AMONG THREE ALGORITHMS

\begin{tabular}{|c||c|c|c|}
\hline$E_{b} / N_{0}(d B)$ & Algorithm 1 & Wu's algorithm & IEED algorithm \\
\hline 7.0 & 1.379 & 36.665 & 2227.033 \\
\hline 8.0 & 1.461 & 30.227 & 2220.900 \\
\hline 9.0 & 1.477 & 26.419 & 2242.067 \\
\hline 10.0 & 1.364 & 23.481 & 2212.733 \\
\hline
\end{tabular}

are considered. From the different tests, We have demonstrated that the newly proposed decoding algorithms provide substantial performance gains when compared with the classic BM algorithm in terms of FER.

In the cases with small $\sigma_{a}$ which correspond to deep fading, Algorithm 1 performs nearly as well as Algorithm 2 despite the fact that the former has no fading location information. Also, Algorithm 1 always outperforms Wu's algorithm and even performs better than the IEED algorithm in some scenarios.

When the parameter $\sigma_{a}$ is relatively large (typically, say $\sigma_{a} \geq 0.4$ ), the IEED algorithm often achieves better performance when compared with Algorithm 1 whereas Algorithm 2 always works very well since the fading locations are assumed to be aforehand known. We also note that the IEED algorithm performs better than our proposed algorithms when the burst length is comparatively short even if $\sigma_{a}$ is small. Nonetheless, it should be highlighted that the IEED algorithm requires a very high computational complexity.

We also observe from the above figures that the performance prediction of the proposed algorithms matches well with the simulations in the deep-fading region. In some cases, the estimated and simulated performance curves are almost indistinguishable. Along with the increase of $\sigma_{a}$, the accuracy of prediction of Algorithm 1 drops gradually while the performance of Algorithm 2 can still be estimated very well.

\section{CONCLUSION}

In this paper, two new soft information based decoding algorithms for RS codes transmitted over the burst Rayleigh fading channels are proposed. They originate from the fact that the capability of correcting erasures is twice as much as that of correcting errors. By treating some erroneous received symbols that satisfy the threshold criteria as erasures, the proposed algorithms significantly outperform the BM algorithm in terms of FER performance with a comparable computational complexity. In particular, the new algorithms exhibit robustness for various code parameters and fading configurations, and achieve a better trade-off when compared with the existing decoding algorithms from error-correcting performance and computational complexity points of view.

Additionally, the FER performance of the proposed decoding algorithms and the BM algorithm over the burst Rayleigh fading channels can be mathematically analyzed, which avoids extensive time-consuming simulations when one is interested in the gain of new algorithms at very low FER, say FER $\leq$ $10^{-8}$. It is expected that the ideas presented in this paper can be generalized to other burst error scenarios. 


\section{ACKNOWLEDGMENT}

The authors would like to thank Prof. Brian M. Kurkoski for his enlightening discussion on the channel model utilized in this paper.

\section{REFERENCES}

[1] Telemetry Channel Coding, Recommendation for Space Data System Standard, CCSDS 101.0-B-3, Blue Book, issue 3, May 1992.

[2] C. Basile et al., "The U.S. HDTV standard the grand," IEEE Spectrum, vol. 32, pp. 36-45, Apr. 1995.

[3] DVD Specifications for Rewritable Disc (DVD-RAM) Part 1, Physical Spec., version 2.0, Sep. 1999.

[4] C. Eklund, R. B. Maarks, K. L. Stanwood, and S. Wang, "IEEE standard 802.16: a technical overview of the wireless MAN air interface for broadband wireless access," IEEE Commun. Mag., vol. 40, no. 6, pp. 98-107, June 2002.

[5] E. R. Berlekamp, Algebraic Coding Theory. New York: McGraw- Hill, 1968.

[6] J. L. Massey, "Shift register synthesis and BCH decoding," IEEE Trans. Inf. Theory, vol. 15, no. 1, pp. 122-127, 1969.

[7] Y. Sugiyama, M. Kasahara, S. Hirasawa, and T. Namekawa, "Amethod for solving key equation for decoding Goppa codes," Inf. Control, vol. 27, pp. 87-99, Feb. 1975.

[8] L. Welch and E. R. Berlekamp, Error correction for algebraic block codes, U.S. Patent 4633470 (1983).

[9] M. Sudan, "Decoding of Reed-Solomon codes beyond the errorcorrection bound," J. Complexity, vol. 13, no. 1, pp. 180-193, 1997.

[10] V. Guruswami and M. Sudan, Improved decoding of Reed-Solomon codes and algebraic-geometry codes, IEEE Trans. Inf. Theory, vol. 45, pp. 1757-1767, Sep. 1999.

[11] R. Koetter and A. Vardy, "Algebraic soft-decision decoding of ReedSolomon codes," IEEE Trans. Inf. Theory, vol. 49, no. 11, pp. 28092825, Nov. 2003.

[12] R. Koetter, J. Ma, A. Vardy, and A. Ahmed, "Efficient interpolation and factorization in algebraic soft-decision decoding of Reed-Solomon codes," Proc. 2003 IEEE Int. Symp. Inf. Theory, Yokohama, Japan, 29 June-4 July, 2003, pp. 365.

[13] R. Koetter, J. Ma, and A. Vardy, "The re-encoding transformation in algebraic list-decoding of Reed-Solomon codes," IEEE Trans. Inf. Theory, vol. 57, no. 2, pp. 633-647, Feb. 2011.

[14] J. Bellorado and A. Kavcic, "Low-complexity soft-decoding algorithms for Reed-Solomon codes-part I: an algebraic soft-in hard-out Chase decoder," IEEE Trans. Inf. Theory, vol. 56, no. 3, pp. 945-959, Mar. 2010.

[15] Y. Wu, "Fast Chase decoding algorithms and architectures for ReedSolomon Codes," IEEE Trans. Inf. Theory, vol. 58, no. 1, pp. 109-129, Jan. 2012.

[16] D. Chase, "A class of algorithms for decoding block codes with channel measurement information," IEEE Trans. Inf. Theory, vol. IT-18, pp. 170182, Jan. 1972.

[17] L. Chen, S. Tang, and X. Ma, "Progressive Algebraic Soft-Decision Decoding of Reed-Solomon Codes," IEEE Trans. Commun., vol. 61, no. 2, pp. 433-442, Feb. 2013.

[18] Obaid Ur-Rehman, Natasa Zivic,"Soft decision iterative error and erasure decoder for Reed-Solomon codes," IET Commun., vol. 8, iss. 16, pp. 2863-2870, Nov. 2014.

[19] J. Chen and P. Owsley, "A burst-error-correcting algorithm for ReedSolomon codes," IEEE Trans. Inf. Theory, vol. 38, pp. 1807-1812, Nov. 1992.

[20] E. Dawson and A. Khodkar, "Burst error-correcting algorithm for ReedSolomon codes," Electron. Lett., vol. 31, pp. 848-849, 1995.

[21] L. Yin, J. Lu, K. B. Letaief, and Y. Wu, "Burst-error-correcting algorithm for Reed-Solomon codes," Electron. Lett., vol. 37, pp. 695-697, 2001.

[22] Yingquan Wu, "Novel Burst Error Correction Algorithms for ReedSolomon Codes," IEEE Trans. Inf. Theory, vol. 58, no. 2, pp. 519-529, Feb. 2012.

[23] Soo-Woong Lee, B. V. K. Vijaya Kumar, "Soft-decision decoding of Reed-Solomon codes using successive error-and-erasure decoding," Proc. 2008 IEEE Global Telecommunication Conference (Globecom 2008), New Orleans, Louisiana, 30 Nov.-4 Dec. 2008, pp. 1-5.

[24] Jing Jin, Tho Le-Ngoc, "RS-coded M-QAM schemes using error-anderasure decoding over wireless fading channels," Proc. 2010 8th Annual Communication Networks and Services Research Conference (CNSR 2010), Montreal, QC, Canada, 11-14 May, 2010, pp. 33-39.
[25] S. Jamali and Tho Le-Ngoc, Coded-Modulation Techniques for fading channels, Kluwer Academic Publishers, 1994.

[26] G. D. Forney, "On decoding BCH codes," IEEE Trans. Inf. Theory, vol. IT-11, no. 4, pp. 549-557, Oct. 1965.

[27] G. Dubney, I.S. Reed, T.K. Truong, and J. Yang, "Decoding the (47, 24, 11) quadratic residue code using bit-error probability estimates," IEEE Trans. Commun., vol. 57, no. 7, pp. 1986-1993, July 2009.

[28] T. K. Truong, I. S. Hsu, W. L. Eastman, and I. S. Reed, "Simplified procedure for correcting both errors and erasures of Reed-Solomon code using Euclidean algorithm," IEE Proceedings E-Computers and Digital Technique, vol. 135, no. 6, pp. 318-324, Nov. 1988.

[29] G. D. Forney, "Generalized minimum distance decoding," IEEE Trans. Inf. Theory, vol. 12, no. 2, pp. 125-131, 1966.

[30] Transcoding/FEC Options and Trade-offs for $100 \mathrm{~Gb} / \mathrm{s}$ Backplane and Copper Cable, IEEE Standard 802.3bj, Nov. 2011.

[31] T. K. Truong, J. H. Jeng, and T. C. Cheng, "A new decoding algorithm for correcting both erasures and errors of Reed-Solomon codes," IEEE Trans. Commun., vol. 51, no. 3, pp. 381-388, March 2003.

[32] T. Wadayama, "An iterative decoding algorithm of low density parity check codes for hidden Markov noise channels," Proc. International Symposium on Information Theory and its Applications (ISITA), Honolulu, Hawaii, U.S.A., Nov. 5-8, 2000 


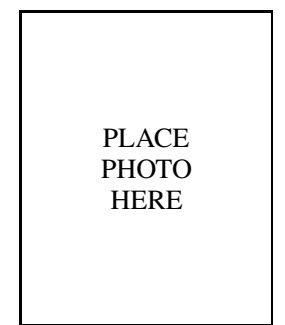

Yong Li (S'11-M'13) received the B.Sc. degree in Electronic and Information Engineering from Chongqing University of Posts and Telecommunications (CQUPT), Chongqing, China, in 2003, the M.S. degree and the Ph.D degree in Communication Engineering from Xiamen University, Fujian, China, in 2006 and 2012, respectively. From May 2018, he joined the College of Computer Science of Chongqing University, where he is currently an associate Professor. From Sep. 2006 to Jan. 2007, he was a research assistant in the department of Electronic Engineering, City University of Hong Kong. From Feb. 2007 to Aug. 2009, he was with Gallop Inc., Chongqing, China. From Sep. 2011 to Aug. 2012, he visited University of California, Davis, USA, as a visiting scholar. From Jan. 2013 to April 2018, he was a faculty member of CQUPT. He has published over 20 papers in peer-reviewed journals or conference proceedings, and held 2 granted national patents. His primary research interests include channel coding, distributed storage coding, and flash memory.

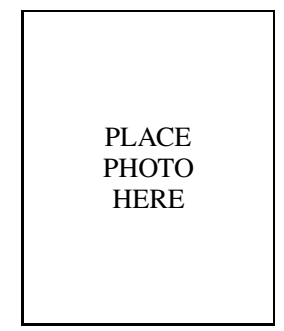

Xiang Huang received the B.Sc. degree in Communication Engineering from Chongqing University of Posts and Telecommunications (CQUPT), Chongqing, China, in 2016, and now he is working towards the M.S. degree in Information and Communication Engineering at CQUPT. His primary research interests include channel coding, wireless communication.

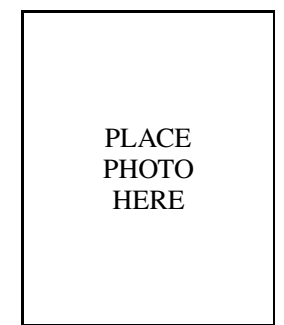

Jiguang He (S'16) received the B.Eng. degree in communications engineering from Harbin Institute of Technology, Harbin, China, in 2010, and M.Sc. degree also in communications engineering from Xiamen University, Xiamen, China, in 2013. From September 2013 to March 2015, he was with Key Laboratory of Millimeter Waves at City University of Hong Kong, conducting research on channel tracking over millimeter wave MIMO systems. Since June 2015, he has been with Centre for Wireless Communications (CWC), Department of Communications Engineering, University of Oulu, Oulu, Finland, studying toward his $\mathrm{Ph} . \mathrm{D}$ degree. His research interests span cooperative communications, network information theory, joint source and channel coding, and distributed compressive sensing.

\begin{tabular}{|c|} 
\\
\\
PLACE \\
PHOTO \\
HERE \\
\end{tabular}

Hongqing Liu (M'15-SM'17) received his Bachelor and Master degrees, from Xidian University, Xi' an Shaanxi, China, in 2003 and 2006, respectively, and $\mathrm{Ph} . \mathrm{D}$. degree from City University of Hong Kong, Hong Kong, China, in 2009, all in electronic engineering. In 2009, after graduation, he joined Acoustic Research Laboratory (ARL), National University of Singapore (NUS), as a research fellow. Since 2013, he has been with School of Communication and Information Engineering, Chongqing University of Posts and Telecommunications (CQUPT), as a faculty member. His research interests lie in the areas of statistical signal processing and convex optimization, including sparse recovery, localization/tracking and parameter estimation.

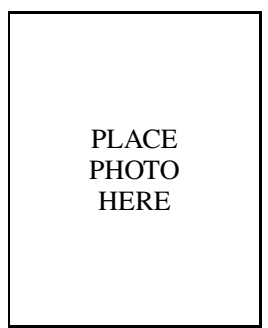

Trieu-Kien Truong (M'82-SM'83-F'99) was born in Vietnam on December 4, 1944. He received the B.S. degree from National Cheng Kung University, Tainan, Taiwan, in 1967, the M.S. degree from Washington University, St. Louis, MO, in 1971, and the Ph.D. degree from the University of Southern California, Los Angeles, in 1976, all in electrical engineering. From 1975 to 1992 , he was a Senior Member of Technical Staff (E6) with the Communication System Research Section, Jet Propulsion Laboratory, Pasadena, CA. Currently, he is a Chair Professor of Collage of Electrical and Information Engineering, I-Shou University, Kaohsiung, Taiwan. His research interests include error-correcting codes, VLSI architecture design, communication systems, signal processing, and image compression. Dr. Troung served as an Editor in the Asia area for the Journal of Visual Communication and Image Representation and as an Editor in the area of Coding Theory and Techniques for the IEEE TRANSACTIONS ON COMMUNICATIONS 